\title{
Serial neuroimaging in infants with abusive head trauma: timing abusive injuries
}

\author{
Clinical article
}

\author{
Ray Bradford, M.D., ${ }^{1}$ Arabinda K. Choudhary, M.D., M.R.C.P., F.R.C.R., ${ }^{1}$ \\ AND Mark S. Dias, M.D. ${ }^{2}$ \\ Departments of ${ }^{1}$ Radiology and ${ }^{2}$ Neurosurgery, Penn State College of Medicine, Penn State \\ Milton S. Hershey Medical Center, Hershey, Pennsylvania
}

\begin{abstract}
Object. The appearance and evolution of neuroimaging abnormalities following abusive head trauma (AHT) is important for establishing the time frame over which these injuries might have occurred. From a legal perspective this frames the timing of the abuse and therefore identifies and excludes potential perpetrators. A previous pilot study involving 33 infants with AHT helped to refine the timing of these injuries but was limited by its small sample size. In the present study, the authors analyzed a larger group of 210 cases involving infants with AHT to chronicle the first appearance and evolution of radiological (CT, MRI) abnormalities.

Methods. All children younger than 24 months admitted to the Penn State Hershey Medical Center with AHT over a 10-year period were identified from a medical record review; the time of injury was determined through an evaluation of the clinical records. All imaging studies were analyzed, and the appearance and evolution of abnormalities were chronicled on serial neuroimaging studies obtained in the days and weeks after injury.

Results. One hundred five infants with specific injury dates and available imaging studies were identified; a subset of 43 children additionally had documented times of injury. In infants with homogeneously hyperdense subdural hematomas (SDHs) on initial CT scans, the first hypodense component appeared within the SDH between 0.3 and 16 days after injury, and the last hyperdense subdural component disappeared between 2 and 40 days after injury. In infants with mixed-density SDHs on initial scans, the last hyperdense component disappeared between 1 and 181 days. Parenchymal hypodensities appeared on CT scans performed as early as 1.2 hours, and all were visible within 27 hours after the injury. Rebleeding into SDHs was documented in 17 cases (16\%) and was always asymptomatic.

Magnetic resonance imaging of the brain was performed in 49 infants. Among those with SDH, 5 patterns were observed. Patterns I and II reflected homogeneous SDH; Pattern I (T1 hyperintensity and T2/FLAIR hypointensity, "early subacute") more commonly appeared on scans performed earlier after injury compared with Pattern II (T1 hyperintensity and T2/FLAIR hyperintensity, "late subacute"), although there was considerable overlap. Patterns III and IV reflected heterogeneous SDH; Pattern III contained relatively equal mixtures having different intensities, whereas Pattern IV had fluid that was predominantly T1 hypointense and T2/FLAIR hyperintense. Again, Pattern III more commonly appeared on scans performed earlier after injury compared with Pattern IV, although there was significant overlap.

Conclusions. These data extend the preliminary data reported by Dias and colleagues and provide a framework upon which injuries in AHT can be timed as well as the limitations on such timing estimates. (http://thejns.org/doi/abs/10.3171/2013.4.PEDS12596)
\end{abstract}

\section{KEY Words • abusive head trauma • shaken baby syndrome • infant traumatic brain injury - magnetic resonance imaging • computed tomography}

A BUSIVE head trauma (AHT) in infants constitutes a serious public health problem both in the United States and worldwide, with an incidence of $15-$ 20 cases per 100,000 person-years among infants $0-24$ months of age and a mortality rate of $15 \%-25 \% .^{2,21,22}$ Clinicians are routinely asked to testify in court as to 1) whether the cranial injuries are consistent with abuse (and inconsistent with an alternative mechanism) and 2)

Abbreviations used in this paper: $\mathrm{ADC}=$ apparent diffusion coefficient; AHT = abusive head trauma; PSHMC = Penn State Hershey Medical Center; $\mathrm{SDH}=$ subdural hematoma. the time frame within which the injuries occurred. Providing a time frame for the injuries may help to identify a potential perpetrator and exclude others. Evidence of repeated injury may also influence the legal judgment. Clinicians who care for these infants must have a good understanding of the scientific literature upon which such timing estimates are made and the methodological limitations of these studies.

Both clinical history and radiological studies can

This article contains some figures that are displayed in color online but in black-and-white in the print edition. 
be used to define a time frame within which trauma has occurred. For example, the literature supports the view that, with rare exceptions, a child suffering disordered breathing/apnea or a serious or lethal traumatic brain injury would be immediately and obviously neurologically impaired from the moment of the injury forward. ${ }^{13,20,34}$ In patients with less severe injuries presenting with a transient loss of consciousness or a single seizure, however, it may be difficult to establish the timing of injury based solely upon the clinical history.

The interpretation of neuroimaging studies (CT, MRI, or both) in the context of injury timing has evolved over the past 2 decades. The previously accepted dogma was that 1) parenchymal brain hypodensities, including "big black brain" and the "reversal sign" (cerebral hemispheres appearing more hypodense than either deep nuclei or posterior fossa structures on CT), are commonly absent on initial imaging studies and routinely appear after 24-48 hours have elapsed;5,35 2) the evolution from acute to chronic subdural hematoma (SDH) requires $1-4$ weeks; ${ }^{1,8,23,35}$ and 3) mixed-density subdural hemorrhages provide prima facie evidence of repeated episodes of AHT. However, these conclusions were derived from studies of conditions other than AHT. For example, the appearance and evolution of parenchymal hypodensities on CT were extrapolated from studies of hypoxic-ischemic encephalopathy (for example, in cases of near drowning); the evolution of acute to chronic subdural hemorrhage was derived from studies in the elderly; and the appearance of blood and blood products within a subdural hemorrhage on MRI was derived largely from studies of intracerebral hematomas. More recent studies have challenged the validity of these concepts and have resulted in an increased appreciation that, for example, hypodensities may appear earlier and mixed-density SDHs may not always reflect seperate episodes of injury.

In 1998, the senior author (M.S.D.) published a study of 33 infants with AHT, chronicling the initial appearance and subsequent temporal evolution of intracranial abnormalities on serial CT scans. ${ }^{9}$ The major conclusions of this study were that 1) parenchymal hypodensities first appear and are conspicuous on the initial CT scans performed an average of 3 hours after the reporting of the injury; 2) acute SDHs are present in $85 \%$ of cases and most commonly (in nearly $80 \%$ of cases) resolve without evolving to chronic SDHs; and 3) when SDHs do evolve from acute to chronic, a hypodense component (with respect to brain) first appears between 3 and 12 days after the injury. However, this study was limited by a small sample size and, in particular, by a limited number of infants (only 4) with an evolving SDH. In an effort to address these limitations, expand upon the findings of the original study, and study both MRI and CT, we undertook a larger study of children with AHT treated at PSHMC.

\section{Methods}

After approval by the Human Subjects Protection Office, the medical records and imaging studies of all infants less than 24 months of age with AHT treated at PSHMC between January 1997 and December 2007 were retrospectively analyzed. Imaging studies performed at other institutions were requested for all children who underwent imaging before transfer to PSHMC. Both the date and, whenever possible, the time of the injury were sought within a reasonable certainty based upon information provided in the medical record, ambulance trip sheets, and emergency department records from referring hospitals. We allowed a degree of uncertainty in the timing of some cases, estimated at plus or minus 1 hour. The computer-generated date and time for each imaging study (CT and MRI) were recorded, and the time between the injury and each study was calculated by subtracting the two times. Since all imaging studies were obtained according to clinical need only, the timing between studies varied from patient to patient. All scans were reviewed by at least 2 of the 3 authors; in no case did we rely only upon the report.

Each CT scan was reviewed systematically to identify both the first appearance of various abnormalities and the evolution of those abnormalities over time. Intracranial CT abnormalities conformed to the definitions used in the previous study. ${ }^{9}$ Subdural hematomas were defined as hemorrhagic fluid collections located under the dura along the convexity, falx, or tentorium. Although a number of studies have confirmed that these hematomas technically lie within the inner dural border zone, the term "subdural" has achieved such widespread acceptance that we refer to these collections as subdural throughout the study. Subdural hematomas were classified as one of 3 types: hyperdense hematomas were uniformly hyperdense with respect to normal brain on $\mathrm{CT}$, hypodense hematomas were uniformly more hypodense than normal brain, and mixed-density collections contained both hyper- and hypodense components. The terms "acute," "subacute," and "chronic" were avoided. Subarachnoid hemorrhage was identified as blood localized within the basal cisterns or sulci; notably, tentorial hemorrhage was defined as subdural rather than subarachnoid in location. Contusions were defined as small punctate hyperdense hemorrhages within the brain parenchyma, and parenchymal hematomas were defined as larger $(\geq 1 \mathrm{~cm})$ parenchymal clots. Edema was defined as a loss of differentiation between gray and white matter. Parenchymal hypodensities were defined as focal or global regions of brain that were hypodense compared with surrounding brain (or in the case of global hypodensities, compared with the density of normal brain). Reversal sign referred to a CT scan on which the cerebral cortex was less dense than the basal ganglia and/or posterior fossa. ${ }^{36,38}$ Encephalomalacia was defined as a focal loss of brain volume, whereas atrophy referred to a global loss of brain volume.

The first appearance of various abnormalities and their evolution over time were documented. For all cases in which the first CT scan showed a uniformly hyperdense SDH, subsequent CT scans were analyzed to determine on which scan and at what time point after the injury 1) a hypodense component first appeared and 2) the hyperdense component completely disappeared. For all cases with mixed-density SDH on the first CT scan, subsequent CT scans were also analyzed to determine the time at which the hyperdense component disappeared. 
This produced a range of times over which these findings (first appearance of hypodense component or last appearance of hyperdense component) could have developed; the maximum time would reflect the time difference between the injury and the first scan to show the finding, whereas the minimum time would be calculated as 1 day after the scan obtained immediately previously. For example, if the first scan to show a hypodense component within an SDH was obtained 10 days after the report of injury and the immediate-previous scan had been obtained 4 days postinjury, the time frame would be 5-10 days.

Abnormalities on MRI scans were similarly documented, although since no infant had more than one MRI scan, the evolution of abnormalities on serial MRI scans could not be studied. MRI sequences included T1-weighted, T2-weighted, FLAIR, gradient echo, diffusion, and ADC sequences. The intensity of SDH was classified as hyperintense or hypointense compared with brain on T1weighted and T2-weighted/FLAIR sequences. Gradient echo sequences were used primarily to identify extraaxial and parenchymal hemorrhages. Parenchymal hypodensities on CT scans corresponded to matched parenchymal T1 hypointensities and T2/FLAIR hyperintensities on MR images. Finally, the diffusion and ADC images were examined to identify areas of parenchyma having matched defects consistent with hypoxic-ischemic injury.

\section{Results}

A total of 210 children younger than 24 months of age were identified, averaging 19 infants per year. Neuroimaging studies could be obtained for 115 children (55\%), of whom 105 had an identifiable date of injury. These 105 cases form the basis of this study. The mean age of the study population was 5.3 months with a median age of 3.9 months and a range of $0.3-23$ months.

\section{Appearance of CT Abnormalities}

The abnormalities on the initial CT scans of these 105 children (Table 1) included SDH in 92\%, skull fractures in $26 \%$, parenchymal hypodensities in $38 \%$, encephalomalacia in $4.8 \%$, and atrophy in $2.9 \%$. In 97 cases, SDH was evident on the initial CT scan; the collections were homogeneously hyperdense in 27 cases $(27.8 \%)$, had mixed density in 56 cases $(57.7 \%)$, and were homogeneously hypodense in 14 cases (14.4\%).

Table 2 lists the average elapsed time between the report of injury and the earliest CT scan on which various intracranial abnormalities first appeared. As expected, acute epidural hematomas, homogeneously hyperdense $\mathrm{SDH}$, subarachnoid hemorrhage, and parenchymal hypodensities were first identified on early scans, whereas atrophy and encephalomalacia first became apparent on later scans. Parenchymal hypodensities appeared most frequently on the initial CT scans performed, on average, 2.6 hours after the report of injury.

\section{Evolution of Subdural Hematomas}

Ninety-seven infants had SDH on the first CT scan; 27 had uniformly hyperdense SDH, 56 had mixed-density
R. Bradford, A. K. Choudhary, and M. S. Dias

TABLE 1: Frequency of intracranial abnormalities on initial CT scans following AHT*

\begin{tabular}{lc}
\hline \multicolumn{1}{c}{ Finding } & No. of Cases $(\%)$ \\
\hline skull fracture & $27(26)$ \\
EDH & $2(1.9)$ \\
SDH & $97(92)$ \\
SAH & $15(14)$ \\
contusion & $14(13)$ \\
hypodensity & $40(38)$ \\
edema & $31(30)$ \\
encephalomalacia & $5(4.8)$ \\
atrophy & $3(2.9)$ \\
\hline
\end{tabular}

* $\mathrm{EDH}=$ epidural hematoma; $\mathrm{SAH}=$ subarachnoid hemorrhage.

$\mathrm{SDH}$, and 14 had homogeneously hypodense SDH on the initial scans (Fig. 1). Of the 27 infants with uniformly hyperdense SDH on initial CT scans, 7 had no further CT scans during the follow-up period; in another 8, the SDH remained hyperdense on the last scan obtained; and in 1 infant, the SDH rebled during follow-up. These 16 cases were excluded from further analysis. The remaining 11 cases were analyzed to determine the time between the report of injury and the first scan to show a hypodense component within the SDH. These times ranged from a minimum of 0.3 to a maximum of 16 days (Fig. 2). For cases 1-6 the interval between scans was less than one day, allowing for more precise timing estimates, whereas the interval between scans for cases 7-11 did not allow such a narrow time estimate. From the first 6 cases, it appears that a hypodense component may appear within a uniformly hyperdense SDH within 1-3 days of the report of injury. The theoretical minimum times ( 1 day after the last CT showing a homogeneously hyperdense SDH) ranged between 1 and 4 days while the maximum documented times ranged from 1 to 16 days (Fig. 2). There were 5 infants for whom serial scans allowed a determination as to the time interval between the injury and the disappearance of the last hyperdense component (Fig. 3). The time frame was between 2 and 40 days; the long interscan intervals precluded a narrower time frame. The theoretical minimum times (1 day after the last CT with a hyperdense component) were between 2 and 8 days, whereas the maximum times were between 19 and 40 days.

Of 56 infants with mixed-density SDH on initial CT scans, 6 underwent surgical drainage, 3 had rebleeding during the follow-up period, and 5 had no subsequent imaging studies; all were excluded from further analysis. Of the remaining 42 infants, 10 had mixed-density SDH on all subsequent CT scans, and 8 had complete resolution of SDH on the follow-up studies. In the remaining 24 infants, the time for the last hyperdense component to disappear ranged from 1 to 181 days (Fig. 4). The minimum theoretical times ( 1 day after the last CT having a hyperdense subdural component) ranged between 1 and 79 days, while the maximum times ranged between 2 and 181 days. 
Serial neuroimaging after abusive head trauma

TABLE 2: Timing of the first appearance of various intracranial abnormalities on first 5 CT scans performed postinjury among 105 patients having identifiable injury dates*

\begin{tabular}{|c|c|c|c|c|c|}
\hline \multirow[b]{2}{*}{ Abnormality } & \multicolumn{5}{|c|}{ Scan } \\
\hline & I & II & III & IV & V \\
\hline EDH & $6.4 \mathrm{hrs}(1)$ & $4.5 \mathrm{hrs}(1)$ & & & \\
\hline acute SDH & $6.4 \mathrm{hrs}(33)$ & 14.9 hrs (9) & & & \\
\hline $\mathrm{SAH}$ & 5.8 hrs (4) & $16.1 \mathrm{hrs}(4)$ & & & \\
\hline brain hypodensity & $2.6 \mathrm{hrs}(8)$ & 16.2 hrs (6) & $62.2 \mathrm{hrs} \mathrm{(4)}$ & $78.8 \mathrm{hrs}(1)$ & \\
\hline edema & $10.1 \mathrm{hrs}(7)$ & $4.3 \mathrm{hrs}(4)$ & 36.4 hrs (1) & 78.6 hrs (1) & \\
\hline contusion & $3.4 \mathrm{hrs}(3)$ & 8.9 hrs (3) & 36 hrs (1) & & \\
\hline atrophy & 2.2 hrs (1) & 21.8 days $(4)$ & 57.8 days $(4)$ & 49.5 days (8) & 15.0 days $(4)$ \\
\hline encephalomalacia & & 32.9 hrs (2) & 82.6 hrs (2) & $65.03 \mathrm{hrs}(5)$ & $5.23 \mathrm{hrs}(2)$ \\
\hline
\end{tabular}

* The numbers in parentheses represent the number of cases for which the abnormality was first detected on the particular CT scan (indicated by Roman numerals at the top). The time values represent the average times after the reporting of injury that these scans were performed.

\section{Rebleeding into Existing Subdural Hematomas}

Rebleeding into an SDH was noted on subsequent CT scans in 17 cases (16\%) and was identified on CT scans performed as early as 0.5 days and as late as 195 days after injury (Fig. 5). Rebleeding into SDH was always small in amount, asymptomatic, and discovered incidentally on CT scans performed during routine follow-up care. Many of these children were either still in the hospital or had previously been removed from their homes and placed into foster care at the time when the rebleeding was discovered.

\section{Parenchymal Abnormalities}

Parenchymal hypodensities were identified in 52 infants with known injury dates; in $40(77 \%)$ of these infants, the hypodensities were apparent on the initial CT scan performed within 24 hours of the injury. Sixteen of these 40 infants had both an identifiable date and an identifiable time for the injury (Fig. 6). Among this group, hypodensities appeared on initial CT scans performed as early as 1.2 hours after the report of injury, and all were evident within 27 hours. Brain edema was present on initial CT scans in 28 infants, 27 of whom had specific dates and times for the reporting of injury. Brain edema also presented most often on early CT scans performed between 1 hour and 5 days after injury; in 17 (63\%) of the 23 cases in which it was observed, brain edema was evident on scans performed within the first 24 hours (Fig. 7).

\section{Appearance of MRI Abnormalities}

Magnetic resonance images were available for review in 49 cases, and an identified date and time were available in 43 of these. Figure 8 illustrates the elapsed time between the injury and the MRI scans in these infants. All 43 infants had $\mathrm{SDH}$, and 5 patterns of SDH were identified (Table 3). Patterns I and II reflected a homogeneous $\mathrm{SDH}$; the SDH in Pattern I (6 infants) was homogeneously T1 hyperintense and T2/FLAIR hypointense (designated

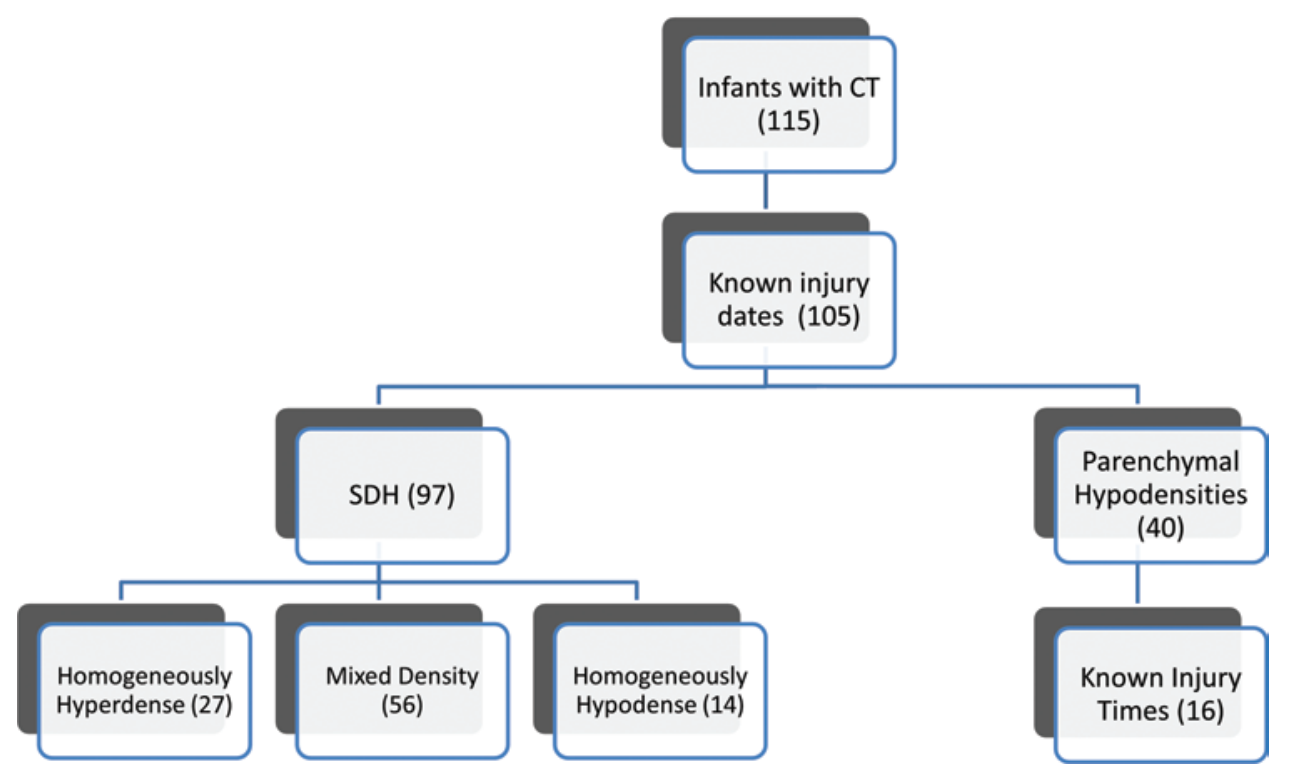

FIG. 1. Distribution of findings on initial CT scans. 


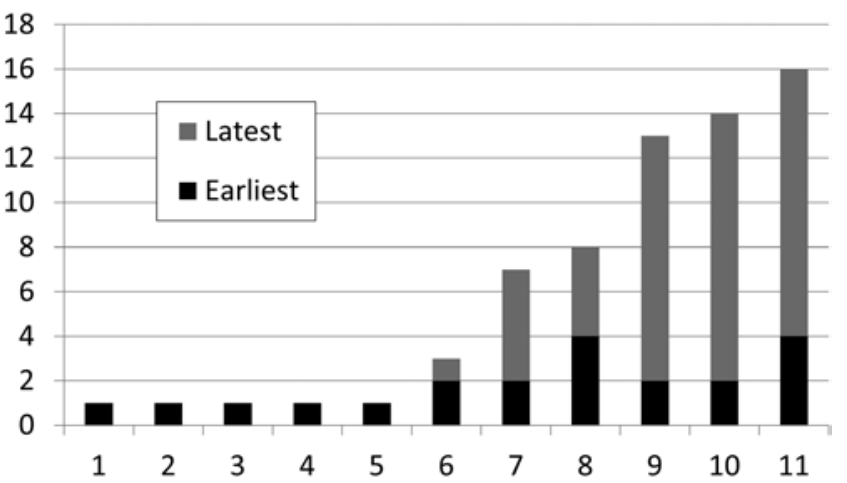

FIG. 2. Time between the report of injury and the first appearance of a hypodense component within an SDH in 11 infants with uniformly hyperdense SDH on the initial CT scan. For each case, there is a range of times over which the hypodense component could have appeared. The earliest times reflect a theoretical minimum time of 1 day after the last scan that had uniformly hyperdense SDH, whereas the latest times reflect the time between injury and the first CT scan to show a hypodense component.

bright/dark) and in Pattern II (6 children) was homogeneously T1 hyperintense and T2/FLAIR hyperintense (designated bright/bright). Radiology textbooks traditionally designate Pattern I as "early subacute" and Pattern II as "late subacute" SDH; the traditional "acute" SDH (T1 isointense and T2/FLAIR hypointense) was not observed in this study. We hypothesized that Pattern I would appear on earlier MRI scans and Pattern II on later scans. In fact, Pattern I did appear more frequently on early MRI scansall but one were present on scans performed during the first 48 hours after injury (Fig. 9 upper). In contrast, Pattern II more often appeared later-two-thirds were present on MRI scans performed later than 72 hours after injury and none were present during the first 24 hours after injury (Fig. 9 upper). However, there was some overlap between the two patterns.

Patterns III and IV reflected mixed-intensity $\mathrm{SDH}$

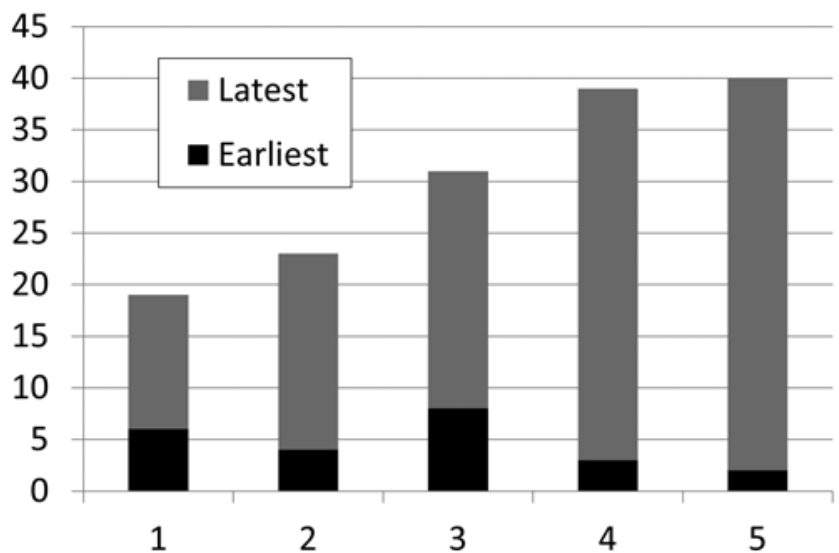

FIG. 3. Time between the report of injury and the last appearance of a hyperdense component within an SDH in 5 infants with uniformly hyperdense SDH on the initial CT scan. For each case, there is a range of times over which the hyperdense component could have disappeared. The earliest times reflect a theoretical minimum time of 1 day after the last scan showing a hyperdense component within the SDH, whereas the latest times reflect the time between injury and the first CT scan to show no hyperdense component.
R. Bradford, A. K. Choudhary, and M. S. Dias

TABLE 3: MRI classification of subdural collections*

\begin{tabular}{lcc}
\hline Type of Subdural Collection & T1 & T2/FLAIR \\
\hline I & $\uparrow$ & $\downarrow$ \\
II & $\uparrow$ & $\uparrow$ \\
III (first component) & $\uparrow$ & $\downarrow$ \\
III (second component) & $\downarrow$ & $\uparrow$ \\
IV (major component) & $\downarrow$ & $\uparrow$ \\
IV (minor component) & $\uparrow$ & $\downarrow$ \\
V & $\downarrow$ & $\uparrow$ \\
\hline
\end{tabular}

${ }^{*} \uparrow=$ Hyperintensity; $\downarrow=$ Hypointensity.

(Fig. 9 lower). In Pattern III (16 children) the SDH contained relatively equal volumes of fluid having two different intensities-one component was $\mathrm{T} 1$ hyperintense and T2/FLAIR hypointense, and the other was T1 hypointense and T2/FLAIR hyperintense (designated mixed bright/ dark and dark/bright respectively). In Pattern IV (14 children) the SDH was of mixed intensity, with most of the collection being T1 hypointense and T2/FLAIR hyperintense and a smaller component being $\mathrm{T} 1$ hyperintense and T2/FLAIR hypointense (designated mixed dark/bright and bright/dark). We hypothesized that Pattern III, having 2 components with relatively equal volumes, would appear on earlier MRI scans compared with Pattern IV, having a predominant dark/bright component that would traditionally be considered "chronic." Again, Pattern III was more frequently present on scans performed within 72 hours of injury, whereas Pattern IV was more commonly present on scans performed after that time. However, there was again significant overlap between the two groups. One final observation was that SDH frequently varied in intensity within different regions of the collection on the same MR image, suggesting that the inhomogeneity may not necessarily be the result of blood products of different ages but simply the inhomogeneous distribution of fluid of different composition within the SDH. Finally, 1 infant had an SDH that was homogeneously T1 hypointense and T2/FLAIR hyperintense (dark/bright) on MR images obtained 26 days after the injury.

Diffusion sequences and ADC maps were obtained in 21 infants, 14 (67\%) of whom had parenchymal abnormalities consistent with ischemia on MRI scans obtained between 0.3 and 5.7 days postinjury. In 8 cases T2/FLAIR hyperintensity was evident in the same distribution, but in 6 cases the abnormality was evident only on the diffusion and ADC sequences. Those children for whom ischemia was identified on only the diffusion/ADC sequences were younger (average age 3.0 months vs 6.1 months) and had earlier postinjury MRI scans (average 1.7 days vs 3.3 days after injury) than those with both diffusion/ADC and T2/ FLAIR changes.

\section{Discussion}

The present study both confirms and extends the findings of the previous study. ${ }^{9}$ The conclusions from these 2 studies are 1) parenchymal abnormalities are apparent on 
Serial neuroimaging after abusive head trauma

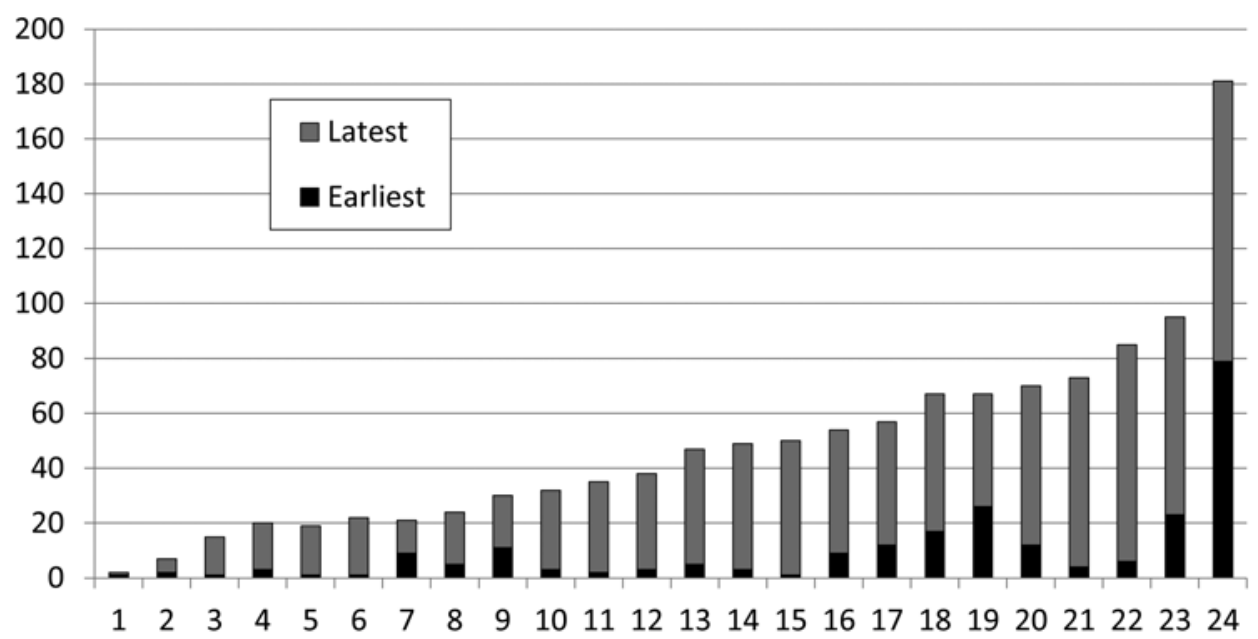

FIG. 4. Time between the report of injury and the last appearance of a hyperdense component within an SDH in 24 infants with mixed-density SDH on the initial CT scan. For each case, there is a range of times over which the hyperdense component could have disappeared. The earliest times reflect a theoretical minimum time of 1 day after the last scan that had some hyperdense component within the SDH, whereas the latest times reflect the time between injury and the first CT scan to show no hyperdense component.

CT scans performed within hours of the reporting of the injury and do not require 24 hours or longer to appear; 2) the evolution of acute to mixed-density SDH may occur much earlier (1-3 days); 3) mixed-density SDH is more common than uniformly hyperdense SDH on initial CT scans and may reflect either prior injuries or an admixture of serum, clotted and unclotted blood, and CSF; and 4) MRI, and especially diffusion weighted imaging, is better than CT for visualizing nonhemorrhagic parenchymal abnormalities and identifying admixtures of subdural blood. While some of these concepts have been increasingly accepted among those who evaluate AHT, the results of these 2 studies provide a framework that both chronicles the changes on neuroimaging after AHT and illustrates the variability of these changes from case to case.

\section{Serial Changes on $C T$}

As has been reported in multiple previous studies, $, 917,27,36,37$ the present study confirms that SDH is the most common intracranial abnormality following AHT

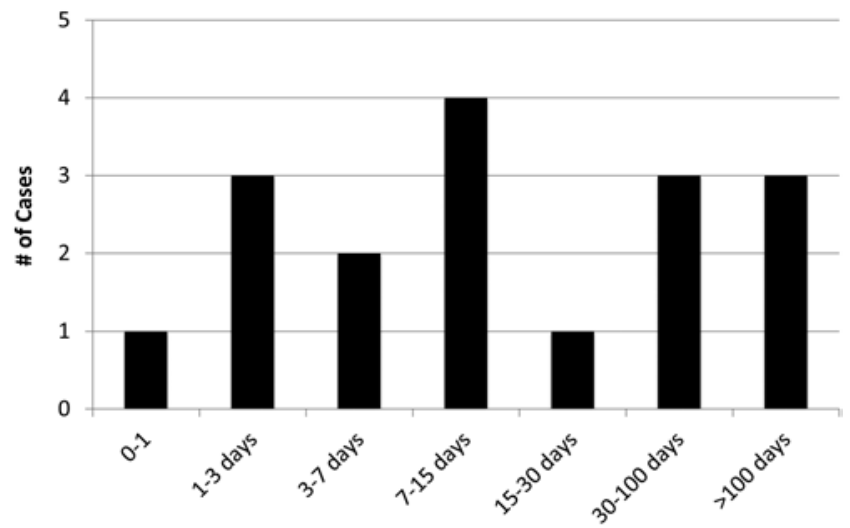

FIG. 5. Time to rebleeding within SDHs. Among 17 infants with documented rebleeding, the time between injury and rebleeding ranged from less than 1 day to 195 days after injury. All infants remained asymptomatic at the time of their subdural rebleeding. (occurring in approximately $80 \%-90 \%$ of children) and is most commonly parafalcine and tentorial in location. Homogeneously hyperdense SDH was present in fewer than one-third of our cases; mixed-density SDH, containing both hyperdense and hypodense components, was present in over half of the infants in the present study, in onethird of children in our prior study, ${ }^{9}$ and in $91 \%$ of infants with AHT in a third study by Vinchon et al. ${ }^{31}$ Mixeddensity SDH in AHT has been interpreted as prima facie evidence of serial injuries, ${ }^{6,25}$ but this concept has recently been challenged. ${ }^{9,11,32,33,39}$ One child in our previous study developed a hypodense subdural collection de novo on a second CT scan performed 19 hours after an initial scan showed no SDH in that region. ${ }^{9}$ In another study of 18 infant victims of motor vehicle crashes with SDH, 3 infants had mixed-density SDH on the initial CT scan. ${ }^{32}$

Although mixed-density SDH has been identified both following accidental and nonaccidental injuries, ${ }^{31,33}$ it appears to be significantly more common following AHT ${ }^{31}$ In a recent study comparing witnessed accidental injuries with confessed cases of AHT, Vinchon and colleagues reported mixed-density SDH on the initial imag-

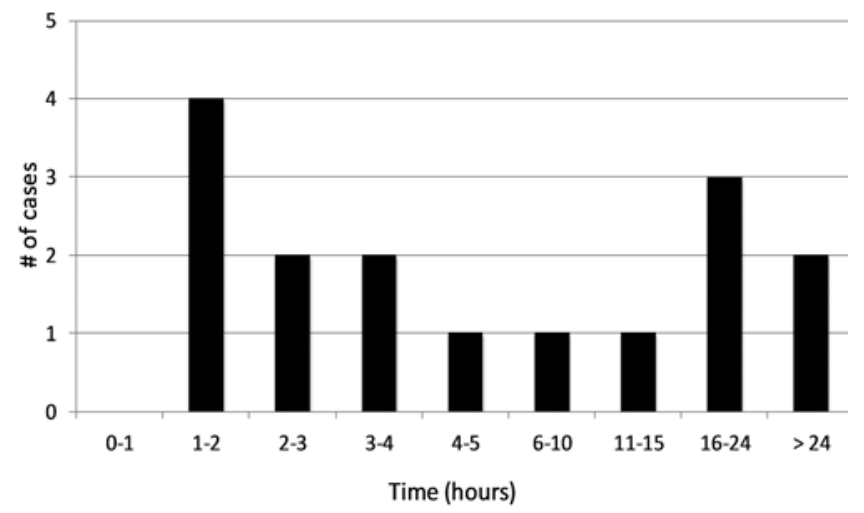

Fig. 6. Time between injury and the first appearance of parenchymal hypodensities on CT. The earliest time interval was 1.2 hours, and all hypodensities were apparent within 27 hours of injury. 


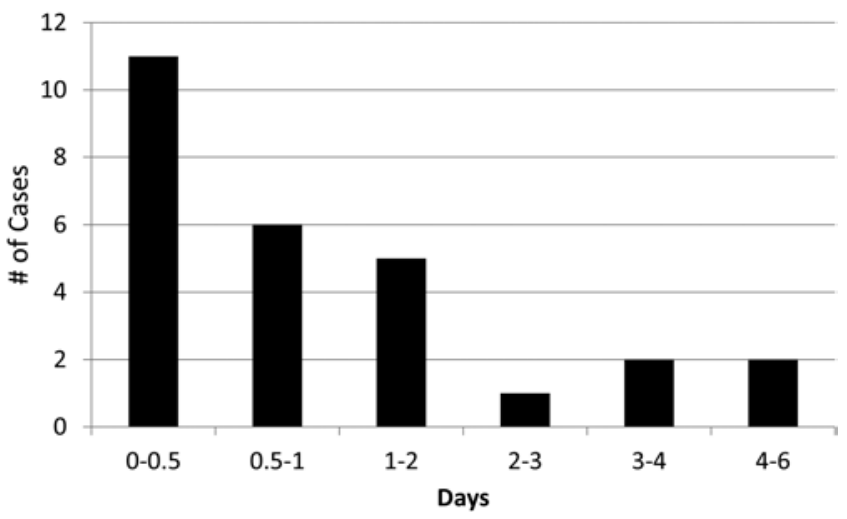

FIG. 7. Time interval between injury and the first appearance of brain edema (loss of gray-white distinction) on CT. The earliest time interval was 1 hour, and in $67 \%$ of the cases, edema was evident on scans performed during the first 24 hours.

ing studies obtained in $53 \%$ of children with accidental injuries and $91 \%$ of children with AHT $(\mathrm{p}<0.001) .{ }^{31} \mathrm{Sev}-$ eral authors have hypothesized that mixed-density SDH may reflect an admixture of fresh clotted blood, unclotted blood or serum, and/or CSF from the subarachnoid space that has migrated into the subdural space through a tear in the arachnoid membrane., ${ }^{9,11,33,39} \mathrm{~A}$ communication between the subarachnoid and subdural spaces in infants with SDH was confirmed in one study by injecting a radioisotope into the CSF via lumbar puncture at the time of subdural drain placement and identifying the radioisotope within the subdural fluid within 3-24 hours. ${ }^{39}$ It is apparent that although mixed-density SDHs are more common after AHT, and some of these likely represent acute and prior injuries, the fact that they are also present early after presumably single accidental injuries makes their interpretation more difficult.

Both the present study and our prior study ${ }^{9}$ establish a rough time frame for the conversion of an acute (homogeneously hyperdense) SDH to a mixed-density and, ultimately, chronic (uniformly hypodense) SDH on CT scans. The accuracy of this time line is unfortunately limited by the amount of time that has elapsed between serial CT scans; subdural hypodensities could have appeared anytime between the day that the hypodensity was first observed on $\mathrm{CT}$ and the day after the last $\mathrm{CT}$ on which

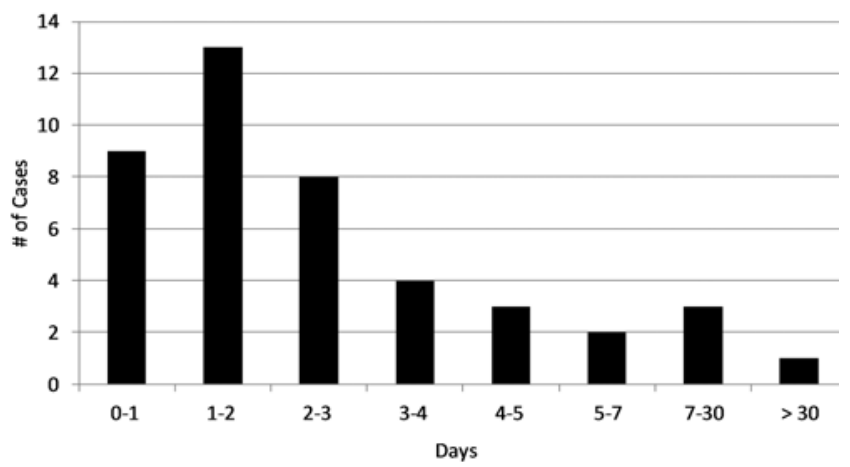

FIG. 8. Time interval between injury and MRI scans in 43 of 49 infants in whom date and time were available. In most cases MRI scans were performed within the first 72 hours.
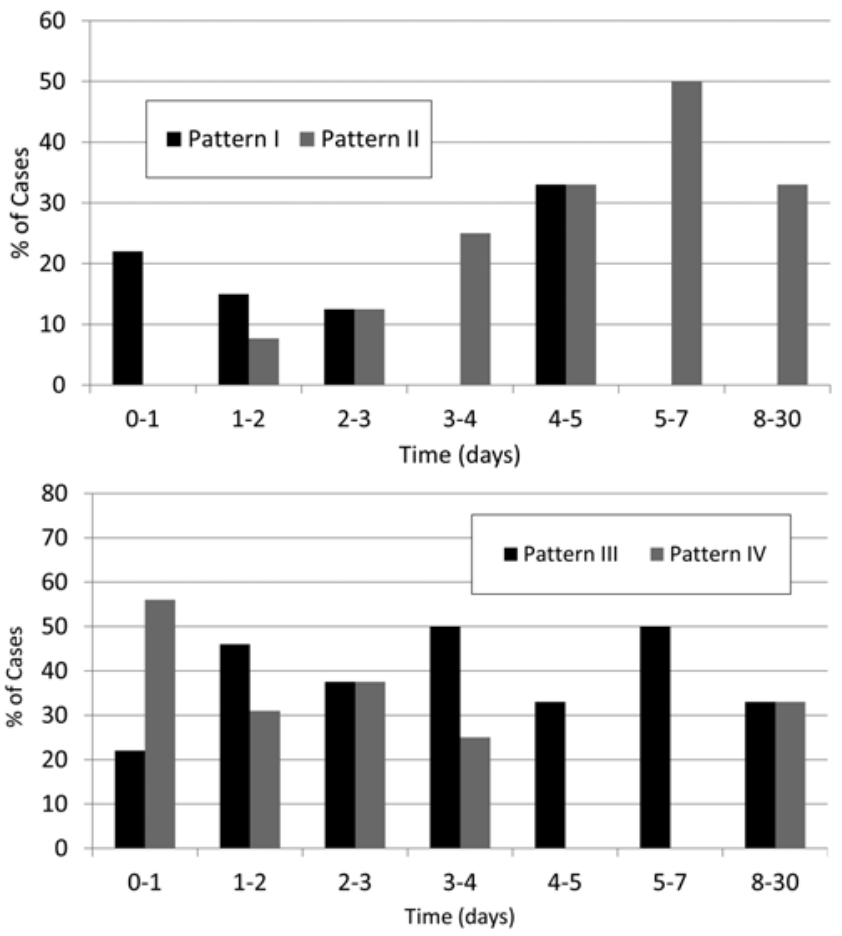

FIG. 9. Timing of MRI patterns in 12 infants with homogeneous SDH. Upper: Pattern I (T1 hypointense and T2/FLAIR hyperintense, traditionally designated "early subacute") appeared earlier than Pattern II (T1 hyperintense and T2/FLAIR hyperintense, traditionally designated "late subacute"), but there was significant overlap. Lower: Timing of MRI patterns in 31 infants with heterogeneous SDH. Pattern III (relatively equal mixtures of 2 components, the first being T1 hyperintense and T2/FLAIR hypointense, and the other T1 hypointense and T2/FLAIR hyperintense. Pattern IV contained predominantly fluid that was T1 hypointense and T2/FLAIR hyperintense and a small amount of fluid that was T1 hyperintense and T2/FLAIR hypointense.

it was absent. Combining the present and prior studies and relying upon those cases where CT scans were more closely spaced (cases 1-6, Fig. 2), subdural hypodensities in infant AHT most commonly appear between the 1 st and 3rd days postinjury, much sooner than the 1-4 weeks accepted previously. The elapsed time for the last hyperdense component to disappear ranged from 2 to 40 days (Fig. 3), but the findings are similarly hampered by the long interscan intervals in some cases, as well as the possibility that subdural rebleeding between scans could have altered the appearance of the SDH. Finally, among the group with mixed SDH on initial scans the elapsed time for the last hyperdense component to disappear was even broader, ranging from a minimum of 2 days to a maximum of 181 days postinjury (Fig. 4). In a smaller study of 20 children with accidental and nonaccidental injuries, some hyperdensity was present in the SDH on all CT scans performed within 1 week postinjury but not on scans performed after Day $11 .{ }^{33}$

\section{Findings on MRI}

It was hoped that MRI might provide a mechanism to better date SDH since the evolution of blood products within intracerebral hematomas has been well studied, ${ }^{14}$ and this schema has been extended to SDH as well. ${ }^{10}$ For 
Serial neuroimaging after abusive head trauma

TABLE 4: Summary of timing of various abnormalities on CT and MRI following AHT*

\begin{tabular}{lccc}
\hline \multicolumn{1}{c}{ Abnormality } & Earliest & Commonly & Longest \\
\hline first hypodense component of SDH & 1 day & $1-8$ days & 16 days \\
last hyperdense component of SDH & 13 days & $20-40$ days & 181 days \\
parenchymal hypodensities & $1 \mathrm{hr}$ & $1-5 \mathrm{hrs}$ & $27 \mathrm{hrs}$ \\
early subacute on MRI & 1 day & $1-3$ days & 5 days \\
late subacute on MRI & 1 day & $2-5$ days & 30 days \\
\hline
\end{tabular}

* Combined results of present and prior study. ${ }^{9}$

intracerebral hematomas, a hyperacute phase (within 12 hours after injury) is identified, during which blood is isointense to brain on T1-weighted images and hyperintense on T2-weighted images, reflecting intracellular oxyhemoglobin. During the acute phase (1-7 days) blood becomes hypointense on both T1- and T2-weighted sequences, reflecting the evolution of intracellular oxyhemoglobin to deoxyhemoglobin. During the early subacute phase (7-13 days) blood becomes hyperintense on T1-weighted images and hypointense on T2-weighted images, reflecting the appearance of intracellular methemoglobin. During the late subacute phase (14-29 days) blood appears hyperintense on both T1- and T2-weighted sequences, reflecting the appearance of extracellular methemoglobin. Finally, in the chronic phase (after 30 days), blood appears hypointense on T1-weighted images and markedly hypointense on T2-weighted images (sometimes referred to as "blooming"), reflecting the appearance of ferritin and hemosiderin. Overall, the MRI appearances of acute and subacute SDH parallel those of intraparenchymal hematomas, but T2 hypointensity ("blooming") is both less frequent and less obvious in chronic SDH than in intraparenchymal hematomas, perhaps because hemosiderin is more readily absorbed from the subdural space, which lacks a blood-brain barrier. ${ }^{10}$

Applying this algorithm to the present study, Pattern I represents an early subacute phase and Pattern II a late subacute phase (Table 4). It is apparent that both of these patterns occur much earlier than they do within intracerebral hematomas, with all Pattern I and many Pattern II SDH appearing on MR images obtained within 7 days of the reporting of injury. Although Pattern I appeared earlier than Pattern II, the overlap makes it difficult to draw any reliable conclusions from the data. Similarly Pattern III (having relatively equal volumes of fluid with dissimilar intensities) appears somewhat earlier after injury than Pattern IV (having predominantly "older"-T1 and T2 hypointense-subdural fluid). Again, however, the extent of the overlap (Fig. 9), the potential that mixed-density SDHs reflect multiple episodes of injury (and therefore ages of blood products), and the possibility of rebleeding between scans make it nearly impossible to draw meaningful conclusions from this analysis. Finally, the recognition that blood of varying intensities can be present in different areas of the SDH on the same MRI scan raises the possibility that the differences in intensity may simply reflect the inhomogeneous distribution of blood products and CSF within the hematoma cavity. In particular, T1hyperintense subdural blood was consistently observed over the occipital convexities even in MRI studies in which the blood elsewhere was hypointense, suggesting that in a recumbent patient, blood products might migrate posteriorly by gravity. A previous study of serial CT scans in children with AHT confirms that, over time, subdural blood following AHT migrates both posteriorly within the interhemispheric fissure and onto the tentorium. ${ }^{33}$

\section{Rebleeding Into Subdural Hematomas}

Finally, rebleeding is a fairly common occurrence after AHT, with a minimum frequency of $15 \%$ in the present study. It is important to emphasize that this rebleeding was small in volume and not associated with acute clinical changes. All cases were discovered on routine imaging studies performed either while the infant was in the hospital or during follow-up clinic visits after the child had been removed from the abusive environment. Although subtle symptoms such as decreased appetite or increased irritability could have been overlooked, we are confident that none of these infants suffered significant neurological decline from subdural rebleeding. Our prior study ${ }^{9}$ had identified a single infant presenting with an acute SDH who had rebleeding 7 days after admission and ultimately underwent surgical evacuation; this single case has been referenced by others as an example of acutely symptomatic subdural rebleeding. ${ }^{16} \mathrm{We}$ want to take this opportunity to clarify that the rebleeding in this infant was asymptomatic; the infant underwent bur-hole drainage 6 days after the rebleeding episode (and 13 days after admission) for a progressively enlarging subacute $\mathrm{SDH}$. Therefore, combining the 2 studies, and after a thorough review of the literature, we cannot find a single infant who has acutely suffered significant neurological deterioration, coma, or death from rebleeding into a preexisting SDH as proposed by Uscinski. ${ }^{29,30}$

\section{Contribution of Neuroimaging to Timing of AHT}

It is apparent from both the present and prior studies that precisely pinpointing the age of an SDH based solely upon imaging findings may be a difficult task. Both the time for an acute SDH to evolve into a mixed-density SDH (first appearance of hypodense component) and the time for a mixed-density SDH to evolve into a chronic SDH (last appearance of hyperdense component) are variable, although broad ranges can be established and the conversion of a hyperdense to mixed-density SDH may occur much more quickly than previously thought. Moreover, since a mixed-density SDH may appear within hours 
after a documented single accidental injury ${ }^{32}$ and additional, asymptomatic rebleeding can occur at any time within a chronic SDH, the mere presence of a hypodense component within an SDH establishes neither that there were repeated injuries nor that a single injury occurred a specific number of days earlier. Although MRI may potentially allow a more accurate means of timing SDH, the limitations of the present study do not allow us to draw firm conclusions at this point.

The present study also demonstrates again that brain abnormalities such as brain edema and parenchymal hypodensities are present and often conspicuous on CT scans performed as early as 1.2 hours and commonly within the first 24 hours after injury. Moreover, diffusion and ADC sequences suggest that these parenchymal hypodensities are ischemic in nature. , $7,18,24,28$ Multiple converging lines of evidence provide support for an ischemic mechanism for brain injuries in AHT. The high incidence of apnea and disordered breathing in AHT has been well documented., ${ }^{9,20}$ In addition, the high frequency and striking persistence of early seizures that follow both AHT and hypoxic-ischemic encephalopathy, ${ }^{9,20}$ but not accidental trauma, point to a unique ischemic-anoxic brain injury mechanism in AHT. Geddes first suggested that the majority of diffuse axonal injuries in fatal cases of AHT are ischemic rather than traumatic based on beta-amyloid precursor protein (BAPP) staining patterns, traumatic axonal injuries being restricted to the cervicomedullary junction and upper spinal cord. ${ }^{12}$ A number of other small case series have documented upper cervical spinal cord injuries in fatal cases of AHT. ${ }^{15,20,26}$ In the largest study to date, Brennan and colleagues documented cervicomedullary and/or upper spinal cord injuries in $29(71 \%)$ of 41 fatal cases of AHT. ${ }^{4}$ The senior author has also observed spinal cord injury on MRI in survivors of AHT (unpublished observations).

This study has several necessary limitations, some of which have already been discussed. First and foremost, it is retrospective, with neuroimaging performed at variable times and based solely upon clinical decisions. Imaging studies were sometimes separated by many days and abnormalities could have developed at any time between the 2 scans; those cases in which scans were performed at shorter time intervals provide a narrower time frame. Second, although this study included larger numbers, breaking the study population into various subgroups reduced the number within each group. Third, the present study relies upon the report of the alleged perpetrator as the only witness to the injury, and this individual, for obvious reasons, may not provide an accurate history. In particular, it is widely suspected that abusive injuries may not immediately be reported to authorities, the perpetrator instead waiting a period of time to see if the child will recover. We recognize, as we did in our prior study, that the precise time of the injury may not coincide with the reported time. Fourth, AHT is always complicated by the possibility of prior injuries; ${ }^{19}$ the presence of mixeddensity SDH in many infants in the study raises this possibility and may influence the nature and evolution of the neuroimaging findings. Lastly, since MRI scans were performed in only a subset of the study population and no child had more than a single MRI, the evolution of changes on MRI could not be studied as CT changes were. To extend our findings further will require a prospective organized study of CT and MRI scans, performed serially on the same children at regular and closely spaced intervals. It may also be helpful to similarly study serial neuroimaging among a population of infants after witnessed accidental injuries with a more reliable time of injury and without prior injuries. However, the low incidence of serious noninflicted intracranial injuries among infants younger than 24 months of age would require a multiinstitutional study, and the findings may not be directly applicable to AHT, given the differences in pathophysiological mechanism(s).

\section{Conclusions}

The present study is the largest such study to date, confirming and extending the findings of our prior smaller study and serving as an important reference for those who are asked to provide a time frame for AHT (Table 4). The combined results from both studies confirm that 1) parenchymal hypodensities on $\mathrm{CT}$ scans commonly arise within hours of injury; 2) mixed-density SDHs are common after AHT; 3) hypodense components may commonly appear de novo within an acute SDH within days after injury, earlier than previously thought; 4) the time required for the disappearance of hyperdense blood within a mixed collection is known only within a broad time frame and requires further study; 5) rebleeding into SDH is a relatively common event, but the quantity is small and, in both studies, the bleeding has been consistently asymptomatic; 6) it is difficult at this point to pinpoint the age of blood within SDH based upon the appearance and interpretation of blood products on various MRI sequences; and 7) MRI scans, particularly diffusion and ADC sequences, may more readily identify parenchymal abnormalities. Our findings suggest that injury timing can usually be broadly established based upon radiological findings, but we again caution against using neuroimaging alone to support overly dogmatic statements. In many cases the clinical history suggests a much more limited temporal window for the injuries within a reasonable probability, and neuroimaging, if it is consistent with this temporal window, can be properly used in conjunction with the clinical history to draw the final conclusions.

\section{Disclosure}

Dr. Dias testifies as an expert witness in child abuse cases and reports receiving non-study related clinical or research support from the Centers for Disease Control, the Pennsylvania Department of Health, and the New York State Children and Family Trust Fund.

Author contributions to the study and manuscript preparation include the following. Conception and design: Dias, Choudhary. Acquisition of data: all authors. Analysis and interpretation of data: all authors. Drafting the article: Dias. Critically revising the article: all authors. Reviewed submitted version of manuscript: all authors. Approved the final version of the manuscript on behalf of all authors: Dias. Study supervision: Dias, Choudhary.

\section{References}

1. Barkovich AJ (ed): Destructive brain disorders of childhood, 
in: Pediatric Neuroimaging, ed 2. New York: Raven Press, 1995, pp 107-176

2. Barlow KM, Minns RA: Annual incidence of shaken impact syndrome in young children. Lancet 356:1571-1572, 2000

3. Biousse V, Suh DY, Newman NJ, Davis PC, Mapstone T, Lambert SR: Diffusion-weighted magnetic resonance imaging in Shaken Baby Syndrome. Am J Ophthalmol 133:249-255, 2002

4. Brennan LK, Rubin D, Christian CW, Duhaime AC, Mirchandani HG, Rorke-Adams LB: Neck injuries in young pediatric homicide victims. Clinical article. J Neurosurg Pediatr 3:232-239, 2009

5. Brown JK, Minns RA: Non-accidental head injury, with particular reference to whiplash shaking injury and medico-legal aspects. Dev Med Child Neurol 35:849-869, 1993

6. Chabrol B, Decarie JC, Fortin G: The role of cranial MRI in identifying patients suffering from child abuse and presenting with unexplained neurological findings. Child Abuse Negl 23:217-228, 1999

7. Chan YL, Chu WCW, Wong GWK, Yeung DKW: Diffusionweighted MRI in shaken baby syndrome. Pediatr Radiol 33: 574-577, 2003

8. Cohen RA, Kaufman RA, Myers PA, Towbin RB: Cranial computed tomography in the abused child with head injury. AJR Am J Roentgenol 146:97-102, 1986

9. Dias MS, Backstrom J, Falk M, Li V: Serial radiography in the infant shaken impact syndrome. Pediatr Neurosurg 29:77-85, 1998

10. Fobben ES, Grossman RI, Atlas SW, Hackney DB, Goldberg HI, Zimmerman RA, et al: MR characteristics of subdural hematomas and hygromas at 1.5 T. AJNR Am J Neuroradiol 153:589-595, 1989

11. Fox RJ, Walji AH, Mielke B, Petruk KC, Aronyk KE: Anatomic details of intradural channels in the parasagittal dura: a possible pathway for flow of cerebrospinal fluid. Neurosurgery 39:84-91, 1996

12. Geddes JF, Hackshaw AK, Vowles GH, Nickols CD, Whitwell HL: Neuropathology of inflicted head injury in children. I. Patterns of brain damage. Brain 124:1290-1298, 2001

13. Gilles EE, Nelson MD Jr: Cerebral complications of nonaccidental head injury in childhood. Pediatr Neurol 19:119-128, 1998

14. Gomori JM, Grossman RI, Goldberg HI, Zimmerman RA, Bilaniuk LT: Intracranial hematomas: imaging by high-field MR. Radiology 157:87-93, 1985

15. Hadley MN, Sonntag VKH, Rekate HL, Murphy A: The infant whiplash-shake injury syndrome: a clinical and pathological study. Neurosurgery 24:536-540, 1989

16. Hymel KP, Jenny C, Block RW: Intracranial hemorrhage and rebleeding in suspected victims of abusive head trauma: addressing the forensic controversies. Child Maltreat 7:329348, 2002

17. Hymel KP, Rumack CM, Hay TC, Strain JD, Jenny C: Comparison of intracranial computed tomographic (CT) findings in pediatric abusive and accidental head trauma. Pediatr Radiol 27:743-747, 1997

18. Ichord RN, Naim M, Pollock AN, Nance ML, Margulies SS, Christian CW: Hypoxic-ischemic injury complicates inflicted and accidental traumatic brain injury in young children: the role of diffusion-weighted imaging. J Neurotrauma 24:106118,2007

19. Jenny C, Hymel KP, Ritzen A, Reinert SE, Hay TC: Analysis of missed cases of abusive head trauma. JAMA 281:621-626, 1999

20. Johnson DL, Boal D, Baule R: Role of apnea in nonaccidental head injury. Pediatr Neurosurg 23:305-310, 1995

21. Keenan HT, Runyan DK, Marshall SW, Nocera MA, Merten DF, Sinal SH: A population-based study of inflicted traumatic brain injury in young children. JAMA 290:621-626, 2003
22. Kesler H, Dias MS, Shaffer M, Rottmund C, Cappos K, Thomas NJ: Demographics of abusive head trauma in the Commonwealth of Pennsylvania. J Neurosurg Pediatr 1:351-356, 2008

23. Levin AV, Magnusson MR, Rafto SE, Zimmerman RA: Shaken baby syndrome diagnosed by magnetic resonance imaging. Pediatr Emerg Care 5:181-186, 1989

24. Parizel PM, Ceulemans B, Laridon A, Ozsarlak O, Van Goethem JW, Jorens PG: Cortical hypoxic-ischemic brain damage in shaken-baby (shaken impact) syndrome: value of diffusionweighted MRI. Pediatr Radiol 33:868-871, 2003

25. Sato Y, Yuh WT, Smith WL, Alexander RC, Kao SC, Ellerbroek CJ: Head injury in child abuse: evaluation with MR imaging. Radiology 173:653-657, 1989

26. Shannon P, Smith CR, Deck J, Ang LC, Ho M, Becker L: Axonal injury and the neuropathology of shaken baby syndrome. Acta Neuropathol 95:625-631, 1998

27. Sinal SH, Ball MR: Head trauma due to child abuse: serial computerized tomography in diagnosis and management. South Med J 80:1505-1512, 1987

28. Suh DY, Davis PC, Hopkins KL, Fajman NN, Mapstone TB: Nonaccidental pediatric head injury: diffusion-weighted imaging findings. Neurosurgery 49:309-320, 2001

29. Uscinski RH: Shaken baby syndrome: an odyssey. Neurol Med Chir (Tokyo) 46:57-61, 2006

30. Uscinski RH, McBride DK: The shaken baby syndrome: an odyssey. II Origins and further hypotheses. Neurol Med Chir (Tokyo) 48:151-156, 2008

31. Vinchon M, de Foort-Dhellemmes S, Desurmont M, Delestret I: Confessed abuse versus witnessed accidents in infants: comparison of clinical, radiological, and ophthalmological data in corroborated cases. Childs Nerv Syst 26:637-645, 2010

32. Vinchon M, Noizet O, Defoort-Dhellemmes S, Soto-Ares G, Dhellemmes P: Infantile subdural hematomas due to traffic accidents. Pediatr Neurosurg 37:245-253, 2002

33. Vinchon M, Noulé N, Tchofo PJ, Soto-Ares G, Fourier C, Dhellemmes P: Imaging of head injuries in infants: temporal correlates and forensic implications for the diagnosis of child abuse. J Neurosurg 101 (1 Suppl):44-52, 2004

34. Willman KY, Bank DE, Senac M, Chadwick DL: Restricting the time of injury in fatal inflicted head injuries. Child Abuse Negl 21:929-940, 1997

35. Zimmerman RA, Bilaniuk LT: Pediatric head trauma. Neuroimaging Clin N Am 4:349-366, 1994

36. Zimmerman RA, Bilaniuk LT, Bruce D, Schut L, Uzzell B, Goldberg HI: Computed tomography of craniocerebral injury in the abused child. Radiology 130:687-690, 1979

37. Zimmerman RA, Bilaniuk LT, Bruce D, Schut L, Uzzell B, Goldberg HI: Interhemispheric acute subdural hematoma: a computed tomographic manifestation of child abuse by shaking. Neuroradiology 16:39-40, 1978

38. Zimmerman RA, Bilaniuk LT, Gennarelli T, Bruce D, Dolinskas C, Uzzell B: Cranial computed tomography in diagnosis and management of acute head trauma. AJR Am J Roentgenol 131:27-34, 1978

39. Zouros A, Bhargava R, Hoskinson M, Aronyk KE: Further characterization of traumatic subdural collections of infancy. Report of five cases. J Neurosurg 100 (5 Suppl Pediatrics): 512-518, 2004

Manuscript submitted December 11, 2012.

Accepted April 29, 2013.

Please include this information when citing this paper: published online June 25, 2013; DOI: 10.3171/2013.4.PEDS12596.

Address correspondence to: Mark S. Dias, M.D., Department of Neurosurgery EC110, Penn State Hershey Medical Center, 30 Hope Dr., Hershey, PA 17033. email: mdias@psu.edu. 